\title{
Anatomía Comparada del Neurocráneo y el Encéfalo de la Raya Mariposa Gymnura micrura (Batoidea: Gymnuridae)
}

\author{
Comparative Anatomy of the Neurocranium and Encephalon of \\ the Butterfly Ray, Gymnura micrura (Batoidea: Gymnuridae)
}

\begin{abstract}
Abraham Kobelkowsky
KOBELKOWSKY,A. Anatomía comparada del neurocráneo y el encéfalo de la raya mariposa Gymnura micrura (batoidea: gymnuridae). Int. J. Morphol., 35(2):644-650, 2017.

RESUMEN: Mediante la comparación del neurocráneo de la "raya mariposa" Gymnura micrura con especies representantes de cinco familias de Batoidea, se reconoce que corresponde al patrón anatómico del orden Myliobatiformes, por la ausencia del rostro, la presencia de una sola fontanela y el mayor desarrollo de los procesos postorbitarios. La especie en estudio difiere de los Urotrygonidae y Dasyatidae por tener los procesos postorbitarios subdivididos. La organización del encéfalo de G. micrura corresponde al patrón anatómico de los Batoidea, sin embargo, a diferencia de los Rhinobatidae, Urotrygonidae y Dasyatidae, el cerebelo es simétrico; en contraste con los Myliobatidae los hemisferios cerebrales son reducidos; y la principal diferencia con relación a los Narcinidae es la ausencia de los lóbulos eléctricos.
\end{abstract}

PALABRAS CLAVE: Elasmobranchii; Cráneo; Cerebro; Nervios craneales; Órganos sensoriales.

\section{INTRODUCCIÓN}

Dentro de la ictiofauna marina mexicana se encuentra la "raya mariposa" Gymnura micrura (Bloch \& Schneider, 1801), la cual en América se distribuye en aguas costeras desde Bahía de Chesapeake, Estados Unidos de Norteamérica hasta Brasil, y en el Golfo de México (McEachran \& de Carvalho, 2003). De acuerdo con Zeade la Cruz et al. (2016) es la especie más importante de elasmobranquios en la captura incidental de la pesquería de camarón en el Golfo de México.

Los estudios morfológicos del género Gymnura son escasos, encontrándose el de Nishida (1990) sobre el neurocráneo de G. japonica, Miyake \& McEachran (1991) sobre el esqueleto y musculatura viscerales, el de GonzálezIsáis \& Domínguez (2004) sobre la musculatura visceral, González-Isáis \& Domínguez sobre G. marmorata; y particularmente de la especie G. micrura el de Kobelkowsky (2004a) sobre su esplacnología, el de Kobelkowsky (2004b) sobre su sistema excretor, y el de Kobelkowsky (2013) sobre su sistema digestivo.

Myagkov (1986), relaciona la morfología del encéfalo de las rayas con aspectos ecológicos y Walker \&
Sherman (2001) describen la anatomía gruesa del encéfalo de Urobatis jamaicensis.

Particularmente sobre G. micrura no se han realizado estudios de su anatomía del neurocráneo, el encéfalo y órganos sensoriales

Considerando la importancia ecológica de la raya mariposa $G$. micrura en las comunidades de peces bentónicos del Golfo de México, el objetivo del presente trabajo es la descripción anatómica de su neurocráneo y encéfalo.

\section{MATERIAL Y MÉTODO}

Se colectaron ejemplares de $G$. micrura mediante una red de arrastre camaronera de prueba en las lagunas costeras de Pueblo Viejo, Tamiahua y Tampamachoco, así como también en el litoral del Golfo de México. Los ejemplares se fijaron en formaldehido al $10 \%$ y se conservaron en alcohol etílico al $70 \%$. 
El análisis anatómico se realizó sobre 10 ejemplares adultos de ambos sexos. Se retiró la piel de las regiones cefálica y branquial, exponiendo dorsalmente tanto el neurocráneo como la musculatura cefálica. En la región ventral se expusieron la musculatura y el esqueleto visceral, los cuales se retiraron con el objeto de observar el neurocráneo.

Se prepararon los neurocráneos mediante descarnado manual y se mantuvieron en alcohol etílico al $70 \%$. El encéfalo, los nervios craneales, los órganos del olfato y los oídos internos se expusieron retirando porciones pequeñas de cartílago del neurocráneo, evitando la ruptura de los nervios craneales.

Se describieron e ilustraron el neurocráneo, el encéfalo, los nervios craneales, los órganos del olfato, los ojos y los oídos internos. Las ilustraciones se realizaron mediante un tubo de dibujo o cámara clara acoplada a un microscopio estereoscópico Wild M3Z.

La terminología del neurocráneo siguió el criterio de Nishida y del encéfalo el de Gilbert (1973).

\section{RESULTADOS}

Neurocráneo. El neurocráneo de G. micrura es deprimido (Fig. 2a) y no presenta rostro. Su borde anterior corresponde a las cápsulas olfatorias. Dorsalmente muestra la amplia fontanela, cuya longitud es cerca de la mitad de la longitud total del neurocráneo. Paralelos a los borde de la fontanela se marcan dos surcos, correspondientes a las ramas supraorbitales del sistema sensorial de la línea lateral (Fig. $2 b)$.

Cada órbita ocular está delimitada por un proceso preorbitario puntiagudo al nivel de las cápsulas olfatorias y un proceso postorbitario (Fig. 2b, c), que a su vez está dividido por una delgada escotadura. Así, el proceso postorbitario tiene una parte triangular y otra cuadrada, entre las que se fija una rama del sistema de la línea lateral en su paso a la unión con el canal supraorbital.

En cada pared del neurocráneo se encuentra un conjunto de forámenes (Fig. 2a), entre los cuales sobresalen el foramen óptico (II), el foramen del trigémino (V) y el foramen del facial (VII). Posterior al foramen óptico se marca una superficie ovalada donde se fija el pedículo óptico, el cual soporta al globo ocular. En el borde inferior y al inicio de la cápsula ótica se encuentra la comisura lateral, detrás de la cual se nota la superficie articular para el cartílago hiomandibular.

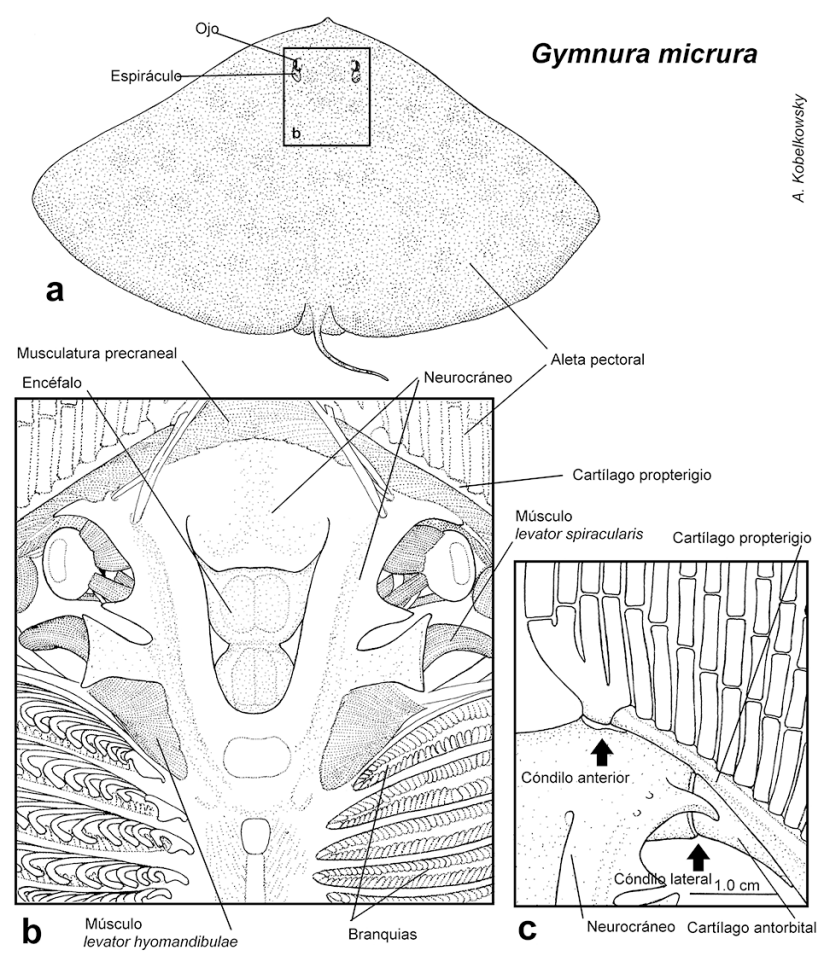

Fig. 1. Localización del neurocráneo y el encéfalo de la raya mariposa Gymnura micrura. a) Vista dorsal de la raya, con el encuadre correspondiente a b. b) Vista dorsal de la región cefálica, después de retirar la piel. c) Vista dorsal de las relaciones articulares (flechas) de la cápsula olfatoria derecha.

En la pared posterior del neurocráneo (Fig. 2 e) se identifica el foramen magnum y a sus lados los cóndilo occipitales, dorsal a los cuales se localizan los forámenes de los nervios vagos $(\mathrm{X})$.

Las cápsulas olfatorias son amplias y muestran ventralmente sus aberturas ovaladas (Fig. 2c), que corresponden a las narinas. Anterior a cada cápsula, se notan los cóndilos anteriores que articulan con el primer radial de cada aleta pectoral (Fig. 1c). Lateral a cada cápsula olfatoria se forma el cóndilo lateral, que articula con el cartílago antorbital (Fig. 1c). Cada foramen olfatorio se localiza dorsal a la cápsula olfatoria, cerca del inicio de la fontanela.

Las cápsulas óticas, las cuales se encuentran en la porción posterior del neurocráneo (Fig. 2b, c) contienen a los oídos internos. En la superficie dorsal, entre ambas cápsulas óticas se reconocen los orificios endolinfáticos (Fig. 2b).

Las órbitas oculares están relativamente reducidas y se encuentran delimitadas por las cápsulas olfatorias y los procesos preorbitarios y postorbitarios (Fig. 2b, c). 


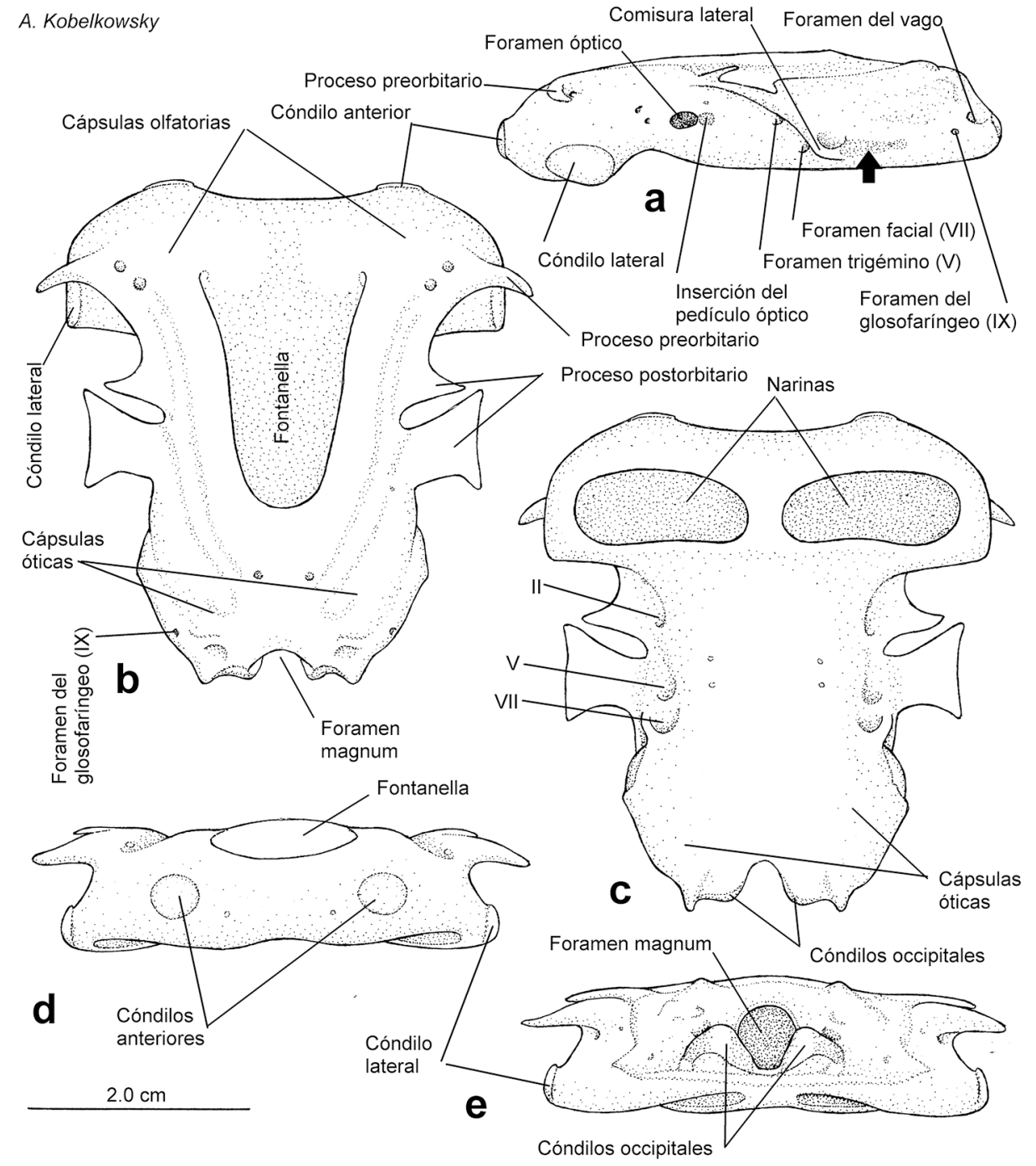

Fig. 2. Neurocráneo de Gymnura micrura. a) Vista lateral izquierda. La flecha señala el área de articulación del cartílago hiomandibular. b) Vista dorsal. c) Vista ventral. d) Vista anterior. e) Vista posterior. siguientes:

Las relaciones topográficas del neurocráneo son las

Los cóndilos anteriores del neurocráneo (Fig. 1c) se articulan con los primeros radiales proximales de las aletas pectorales; los cóndilos laterales (Fig. 1c) se articulan con los cartílagos antorbitales; los cóndilos occipitales ( Fig. 2e) se articulan con el cartílago sinarcual; los canales supraorbitales del sistema de la línea lateral se localizan sobre el techo del neurocráneo, de manera paralela a la fontanela (Fig. 1b, 2b).

La musculatura precraneal se origina de la parte anterior de las cápsulas olfatorias y se inserta en la porción anterior de los cartílagos propterigios (Fig. 1b); los múscu- los extraoculares se originan de la pared de las órbitas oculares y se insertan en los globos oculares (Fig. 3) y están inervados por los nervios craneales III (oculomotor), IV (troclear) y VI (abducente); los músculos spiracularis (Fig. 1b) se originan de las paredes de las cápsulas óticas y se insertan en los cartílagos espiraculares; los músculos levatores hyomandibulae (Fig. 1b) se originan del techo de las cápsulas óticas y se insertan en los cartílagos hiomandibulares.

Porciones de los canales infraorbitales del sistema de la línea lateral hacen contacto con las cápsulas olfatorias; y una de las ramas del canal supraorbital se ubica entre las dos porciones de los procesos postorbitarios del neurocráneo. 


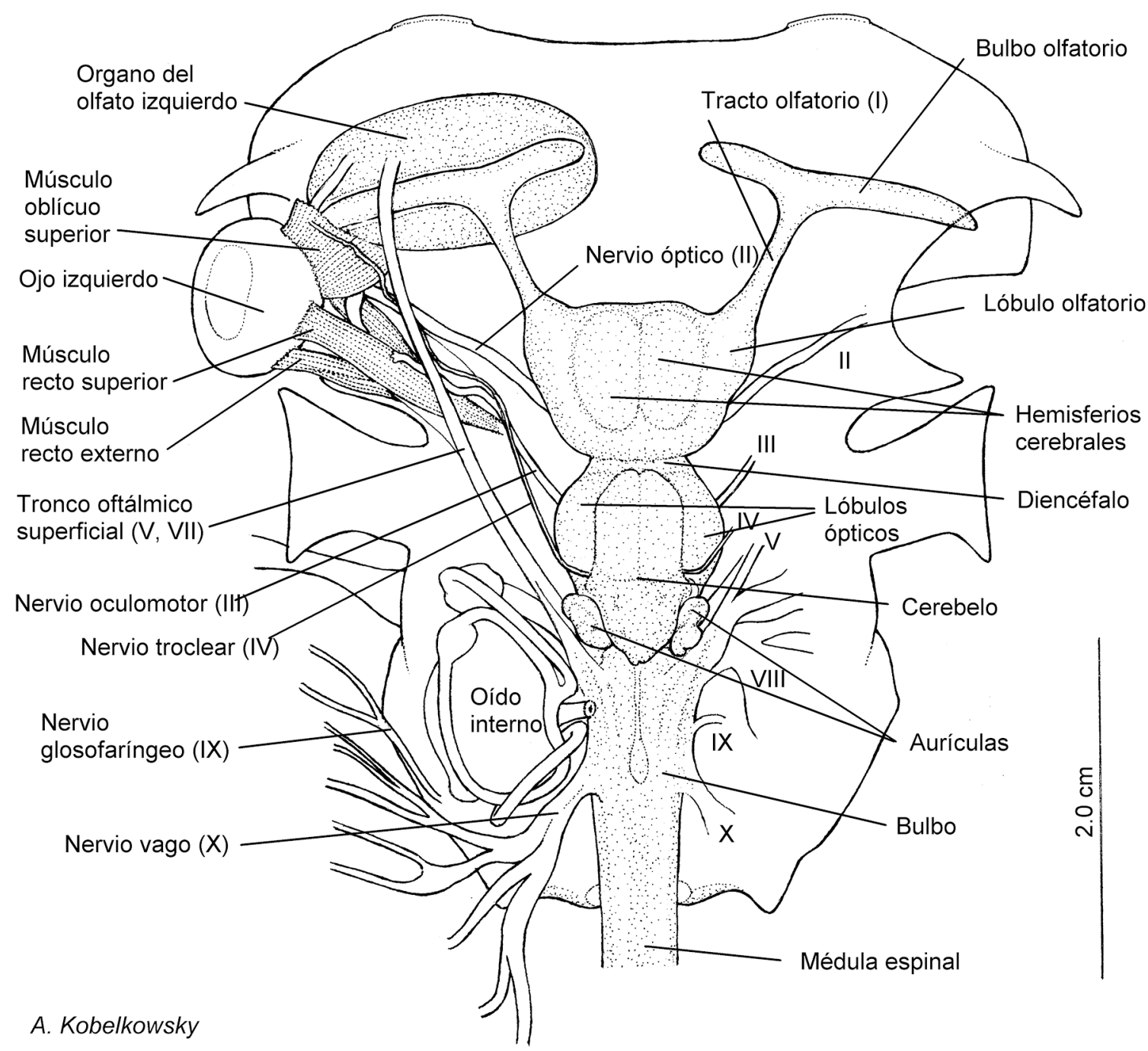

Fig. 3. Encéfalo de Gymnura micrura. Vista dorsal. Los órganos sensoriales del lado derecho están removidos.

Encéfalo. El encéfalo de G. micrura (Fig. 3) es deprimido y se divide en el prosencéfalo, mesencéfalo y rombencéfalo, de los cuales el primero es el más voluminoso y el mesencéfalo el más reducido.

El prosencéfalo se subdivide en el telencéfalo y el diencéfalo. El telencéfalo es relativamente grande; los hemisferios cerebrales son voluminosos y tienen el techo plano. De cada hemisferio sobresale un lóbulo olfatorio, que se continúa hacia delante como tracto olfatorio (nervio olfatorio) y que termina formando el bulbo olfatorio. Los bulbos olfatorios tienen dos porciones desiguales que se adosan al techo del órgano olfatorio.

El diencéfalo es relativamente corto y angosto y ventralmente comprende el quiasmo óptico, los lóbulos in- feriores del infundíbulo, los sacos vasculares y la hipófisis. El quiasma óptico es el contacto de los dos nervios ópticos (II), los cuales son notablemente delgados y largos.

El mesencéfalo está formado por los lóbulos ópticos y el tegmentum. Los primeros son ovalados y están parcialmente cubiertos por el cerebelo. Los nervios trocleares (IV) salen dorsalmente entre los lóbulos ópticos y el cerebelo e inervan los músculos oblicuos superiores. El tegmentum es ventral y del mismo salen los dos nervios oculomotores (III) que inervan los músculos oblicuos inferiores, rectos superiores, rectos inferiores, y rectos inferiores.

El rombencéfalo se subdivide en el cerebelo (metencéfalo) y la médula oblonga (mielencéfalo). El cerebelo es deprimido y tiene una porción anterior rectangular, 
que cubre parcialmente al mesencéfalo y otra posterior triangular que cubre parte de la médula oblonga y que forma las aurículas.

La médula oblonga o bulbo raquídeo es alargada y recta y muestra en su techo la tela coroidea, en forma de una hendidura media dorsal.

De la parte antero-lateral de la médula oblonga salen los nervios trigéminos (V), faciales (VII) y auditivos (VIII); de su superficie ventral salen los nervios abducentes (VI) que inervan los músculos rectos internos, y de su porción postero-lateral salen los nervios glosofaríngeos (IX) y vagos $(\mathrm{X})$.
Órganos sensoriales. El sistema olfatorio de G. micrura está formado por las narinas, los cartílagos nasales, los órganos olfatorios, los bulbos olfatorios, los tractos olfatorios y los lóbulos olfatorios. Cada órgano del olfato contiene numerosas láminas olfatorias (Fig. 4).

El sistema óptico está formado por los globos oculares (Fig. 4), los pedículos ópticos, los músculos extraoculares, los nervios ópticos y el quiasma óptico.

El sistema estato-acústico está formado por la fosa endolinfática, los orificios endolinfáticos y perilinfáticos y los oídos internos (Fig. 3).

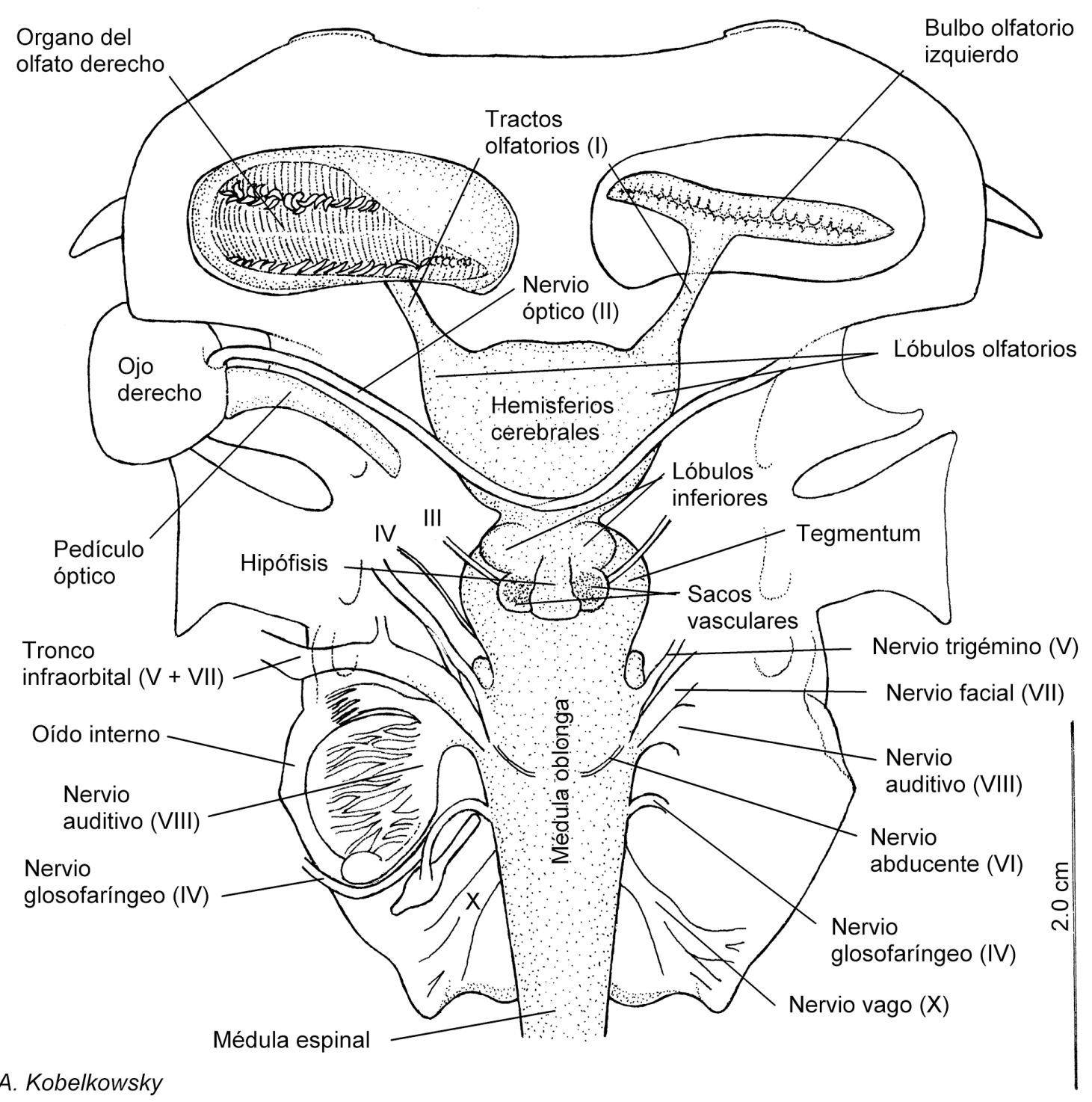

Fig. 4. Encéfalo de Gymnura micrura. Vista ventral. Los órganos de los sentidos del lado izquierdo del ejemplar están removidos, así como también los músculos extraoculares. 
De acuerdo con la Tabla I se reconocen las siguientes diferencias anatómicas del neurocráneo y el encéfalo de G. micrura con relación a especies representantes de las familias Narcinidae (Narcine brasiliensis), Rhinobatidae (Rhinobatos lentiginosus), Rajidae (Raja texana), Urotrygonidae (Urotrygon chilensis) y Dasyatidae ( Dasyatis sabina):

a) Mientras que en G. micrura el neurocráneo no está prolongado en un rostro, el de $N$. brasiliensis lo desarrolla de manera amplia y el de $R$. lentiginosus y $R$. texana es angosto. b) El neurocráneo de G. micrura forma una fontanela, carácter compartido con $U$. chilensis y D. sabina, mientras que $N$. brasiliensis, $R$. lentiginosus y $R$. texana tienen dos fontanelas. c) Los procesos postorbitarios se desarrollan en G. micrura, U. chilensis y D. sabina, mientras que en las restantes especies estudiadas son imperceptibles, sin embargo, dichos procesos en G. micrura están divididos en dos partes por una rama del sistema sensorial de la línea lateral. d) La separación de las aberturas nasales del neurocráneo de $G$. micrura es mayor que en las especies de $R$. lentiginosus y $R$. texana, y es similar a la de $N$. brasiliensis. e) El encéfalo de $G$. micrura tiene una forma similar al de las restantes especies de batoideos considerados en el presente estudio, sin embargo, difiere notablemente del de $U$. chilensis y D. sabina, las cuales tienen el cerebelo notablemente lobulado. Mientras que $R$. lentiginosus y $R$. texana tienen los bulbos olfatorios notablemente asimétricos, G. micrura los presenta casi simétricos; asimismo, son diferentes de los de $N$. brasiliensis por presentarlos trilobulados. Mientras que $R$. lentiginosus tiene los tractos olfatorios notablemente gruesos, G. micrura y las restantes especies en estudio los tiene delgados. f) La principal diferencia de G. micrura, así como también de $R$. lentiginosus, $R$. texana, $U$. chilensis y $D$. sabina con relación a $N$. brasiliensis es la ausencia de los lóbulos eléctricos.

Tabla I. Caracteres diferenciales del neurocráneo y el encéfalo de Narcine brasiliensis (Narcinidae), Rhinobatos lentiginosus (Rhinobatidae), Raja texana (Rajidae), Urotrygon chilensis (Urotrygonidae), Dasyatis sabina (Dasyatidae) y Gymnura micrura (Gymnuridae).

\begin{tabular}{|c|c|c|c|c|c|c|}
\hline & $\begin{array}{l}\text { Narcine } \\
\text { brasiliensis }\end{array}$ & $\begin{array}{l}\text { Rhinobatos } \\
\text { lentiginosus }\end{array}$ & $\begin{array}{l}\text { Raja } \\
\text { texana }\end{array}$ & $\begin{array}{l}\text { Urotrygon } \\
\text { chilensis }\end{array}$ & $\begin{array}{l}\text { Dasyatis } \\
\text { sabina }\end{array}$ & $\begin{array}{l}\text { Gymnura } \\
\text { micrura }\end{array}$ \\
\hline Presencia de rostro & $\begin{array}{l}\text { Amplio y } \\
\text { ramificado }\end{array}$ & Largo y delg ado & Largo y delgado & Ausente & Ausente & Ausente \\
\hline Número de fontanelas & 2 & 2 & 2 & 1 & 1 & 1 \\
\hline $\begin{array}{l}\text { Procesos } \\
\text { post orbitarios }\end{array}$ & Ausentes & Reducidos & Reducidos & $\begin{array}{l}\text { Prolongados } \\
\text { en filamentos }\end{array}$ & Presentes & Subdivididos \\
\hline Aberturas olfatorias & Cercanas entre sí & $\begin{array}{l}\text { Separadas } \\
\text { entre sí }\end{array}$ & $\begin{array}{l}\text { Separadas entre } \\
\text { sí }\end{array}$ & $\begin{array}{l}\text { Cercanas } \\
\text { entre sí }\end{array}$ & Cercanas entre sí & Cercanas entre sí \\
\hline Bulbos olfatorios & $\begin{array}{l}\text { Simétricos y } \\
\text { trilobul ados }\end{array}$ & $\begin{array}{l}\text { Asimétricos y } \\
\text { amplios }\end{array}$ & $\begin{array}{l}\text { Asimétricos y } \\
\text { amplios }\end{array}$ & $\begin{array}{l}\text { Simétricos y } \\
\text { amplios }\end{array}$ & $\begin{array}{l}\text { Simétricos y } \\
\text { amplios }\end{array}$ & $\begin{array}{l}\text { Asimétricos y } \\
\text { angostos }\end{array}$ \\
\hline Tractos olfatorios & Delgados y largos & Gruesos y cortos & Presentes & Presentes & Gruesos y cortos & Delgados \\
\hline Simetría del cerebelo & Presente & Ausente & Presente & Ausente & Ausente & Presente \\
\hline Lobulación del & Discreta & Discreta & Discreta & Notable & Notable & Dis creta \\
\hline Lóbulos eléctricos & Presentes & Ausentes & Ausentes & Ausentes & Ausentes & Ausentes \\
\hline
\end{tabular}

\section{DISCUSIÓN}

La organización morfológica del neurocráneo de la raya mariposa Gymnura micrura corresponde al patrón general de los Elasmobranchii (elasmobranquios) y en particular al de los Batoidea (batoideos). Dentro de estos, corresponde al orden de los Myliobatiformes, por la ausencia del rostro, la presencia de los procesos postorbitarios y la ausencia del arco yugal.

El neurocráneo de la especie en estudio difiere del de Gymnura japonica descrito por Nishida y del de Gymnura marmorata descrito por González-Isáis \& Domínguez (2004) por la forma de la fontanela y el número de foramina del denominado por Nishida canal preorbital.
La organización anatómica del encéfalo de $G$. micrura corresponde al patrón general de los elasmobranquios, como el descrito por Gilbert en el cazón Squalus acanthias, y en particular con el patrón general de los batoideos, descrito por Myagkov en Torpedo marmorata, Raja clavata, Rhinobatos annulatus, Dasyatis sabina, Dasyatis americana, Hexatrygon bickelli, Platyrhinoides triseriata y Urolophus giganteus.

Northcutt (1977) comenta que las rayas tienen una organización compleja del telencéfalo, caracterizada por la reducción de los ventrículos laterales, y de acuerdo con Lisney et al. (2008) existe una correlación del tamaño del 
encéfalo de las rayas y su organización con la filogenia. Así, los Torpediniformes y los Rajiformes tienden a tener el encéfalo relativamente pequeño, con el telencéfalo reducido, la médula oblonga grande y el cerebelo sin foliación, mientras que los Myliobatidae tienen el encéfalo grande, con el telencéfalo voluminoso, el cerebelo notablemente foliado y la médula oblonga reducida.

Myagkov clasifica a los batoideos de acuerdo con la organización de su encéfalo. En un primer grupo incluye a los Rajiformes, con el menor nivel de organización, que implica la forma simple del encéfalo, la simetría del cerebelo, y un buen desarrollo de la médula oblonga; los Torpediniformes tienen el encéfalo similar a de los Rajiformes, sin embargo, presentan lóbulos eléctricos como describen Montes et al. (2014), los cuales están relacionados con los órganos eléctricos; los Dasyatidae muestran el cerebelo grande, asimétrico y circunvolucionado; los Rhinobatidae se encuentran en una situación intermedia. El mismo autor señala que la organización más compleja del cerebelo corresponde a los batoideos que tienen la mayor actividad motora y los hábitos depredadores, lo cual coincide con las observaciones de Yopak et al. (2007) en tiburones. Asimismo, Csilla (2011) reconoce a Manta borostris como el batoideo con mayor masa cerebral, mientras que Mobula japanica es la que tiene mayor masa telencefálica.

Comparativamente con especies representantes de una familia de cada uno de los órdenes de Batoidea, se identifica como el carácter del neurocráneo exclusivo de $G$. micrura a la subdivisión de los procesos postorbitarios. Dicha subdivisión de aspecto de ranura, permite el paso de una de las ramas del sistema sensorial de la línea lateral, para su unión con la rama supraorbital.

La ausencia del rostro en las tres especies de batoideos estudiadas ( $U$. chilensis, D. sabina y G. micrura) se considera un carácter del orden Myliobatiformes.

KOBELKOWSKY, A. Comparative anatomy of the neurocranium and encephalon of the butterfly ray, Gymnura micrura (Batoidea: Gymnuridae). Int. J. Morphol., 35(2):644-650, 2017.

SUMMARY. By comparing the neurocranium of the "butterfly ray" Gymnura micrura with representative species of five families of Batoidea, it is recognized that it corresponds to the anatomical pattern of the order Myliobatiformes, because of the absence of the rostrum, the presence of a single fontanelle and the development of the postorbital processes. The species under study differs from the Urotrygonidae and Dasyatidae by having subdivided postorbital processes. The organization of the encephalon of G. micrura corresponds to the anatomical pattern of the Batoidea. However, unlike the Rhinobatidae, Urotrygonidae, and Dasyatidae, the cerebellum is symmetrical; in contrast with the Myliobatidae, the cerebral hemispheres are reduced; and, the main difference in relation to the Narcinidae, is the absence of electrical lobes.

KEY WORDS: Elasmobranchii; Cranium; Brain; Cranial nerves; Sensory organs.

\section{REFERENCIAS BIBLIOGRÁFICAS}

Csilla, A. Encephalization and brain organization of mobulid rays (Myliobatiformes, Elasmobranchii) with ecological perspectives. Open Anat. J., 3:1-13, 2011

Gilbert, S. G. Pictorial Anatomy of the Dogfish. Seattle, University of Washington Press, 1973. pp.59.

González-Isáis, M. \& Domínguez, H. M. Comparative anatomy of the superfamily Myliobatoidea (Chondrichthyes) with some comments on phylogeny. J. Morphol., 262(1):517-35, 2004.

Kobelkowsky, A. El sistema excretor de la raya mariposa Gymnura micrura (Pisces: Rajiformes). An. Inst. Biol. Univ. Nac. Auton. Mex. Ser. Zool., 75(1):185-92, 2004b

Kobelkowsky, A. Esplacnología de la raya mariposa Gymnura micrura (Rajiformes: Gymnuridae). Vertebr. Mex., 14:1-8, 2004a.

Kobelkowsky, A. Morfología del sistema digestivo de la raya mariposa Gymnura micrura (Batoidea: Gymnuridae). Bol. Investig. Mar. Cost., 42(1):57-71, 2013

Lisney, T. J.; Yopak, K. E.; Montgomery, J. C. \& Collin, S. P. Variation in brain organization and cerebellar foliation in chondrichthyans: batoids. Brain Behav. Evol., 72(4):262-82, 2008.

McEachran, J. D. \& de Carvalho, M. R. Gymnuridae. Butterfly Rays. En: Carpenter, K. E. (Ed.). The Living Marine Resources of the Western Central Atlantic. Roma, FAO, 2003. pp.575-7.

Montes, D. H. M.; López, B. R. \& González, I. M. Morphological Study of the Brain and Cranial Nerves of Diplobatis ommata (Elasmobranchii: Narcinidae). Int. J. Morphol., 32(4):1152-5, 2014.
Myagkov, N. A. Brain structure of skates in relation to their ecology. Neurosci. Behav. Physiol., 16(4):356-62, 1986.

Nishida, K. Phylogeny of the order Myliobatoidei. Mem. Fac. Fish. Hokkaido Univ., 37:1-108, 1990

Northcutt, R. G. Elasmobranch central nervous system organization and its possible evolutionary significance. Am. Zool., 17(2):411-29, 1977.

Walker, B. K. \& Sherman, R. L. Gross brain morphology in the yellow stingray, Urobatis jamaicensis. Fla. Sci., 64(4):246-9, 2001.

Yopak, K. E.; Lisney, T. J.; Collin, S. P. \& Montgomery, J. C. Variation in brain organization and cerebellar foliation in chondrichthyans: sharks and holocephalans. Brain Behav. Evol., 69(4):280-300, 2007.

Zea-de la Cruz, H.; Oviedo-Pérez, J. L.; Balderas-Telles, J.; Acosta-Barbosa, G.; Balderas-Ramírez, L. A. \& Balam-Ché, L. I. Abundancia y estructura poblacional de la raya mariposa Gymnura micrura asociadas al arrastre camaronero en el Golfo de México. Cienc. Pesq., 24(1):15-26, 2016.

\section{Dirección para correspondencia:}

Abraham Kobelkowsky

Laboratorio de Peces

Departamento de Biología

Universidad Autónoma Metropolitana, Unidad Iztapalapa.

Av. San Rafael Atlixco 186.

Col. Vicentina. Iztapalapa. 09340

Ciudad de México - MÉXICO

Recibido : 24-12-2016

Aceptado: 02-03-2017

E-mail: akd@xanum.uam.mx 